\title{
A universal method to easily design tough and stretchable hydrogels
}

\author{
Chisa Norioka', Yuino Inamoto', Chika Hajime', Akifumi Kawamura ${ }^{1,2}$ and Takashi Miyata (1) ${ }^{1,2}$
}

\begin{abstract}
Hydrogels are flexible materials that have high potential for use in various applications due to their unique properties. However, their applications are greatly restricted by the low mechanical performance caused by high water content and inhomogeneous networks. This paper reports a universal strategy for easily preparing hydrogels that are tough and stretchable without any special structures or complicated processes. Our strategy involves tuning the polymerization conditions to form networks with many polymer chain entanglements to achieve energy dissipation. Tough and stretchable hydrogels can be prepared by free radical polymerization with a high monomer concentration and low cross-linker content to optimize the balance between physical and chemical cross-links by entanglements and covalent bonds, respectively. The strategy of using polymer chain entanglements for energy dissipation allows us to overcome the limitation of low mechanical performance, which leads to the wide practical use of hydrogels.
\end{abstract}

\section{Introduction}

Hydrogels are soft materials that consist of physically or chemically cross-linked polymer networks and a large quantity of water. Hydrogels have a high water content and low elastic modulus ( 100 kPa) and exhibit stimulusresponsive behavior, similar to biological tissues; thus, hydrogels have many potential applications as biomaterials for drug delivery systems, biosensors, and cell culture ${ }^{1-5}$. Although hydrogels are soft and flexible, they are also weak and brittle ${ }^{6}$. Standard hydrogels can be easily broken when subjected to a sub-MPa tensile stress or large deformation. The fracture energy of hydrogels is only approximately one hundredth that of biological tissues such as cartilage e $^{7,8}$. Recently, to overcome the low mechanical properties of hydrogels, some researchers have strategically designed network structures of hydrogels, such as sliding-ring hydrogels $^{9,10}$, nanocomposite hydrogels ${ }^{11}$, double network (DN) hydrogels $^{12-14}$, and other structures ${ }^{15-22}$. DN hydrogels,

Correspondence: Takashi Miyata (tmiyata@kansai-u.ac.jp)

${ }^{1}$ Department of Chemistry and Materials Engineering, Kansai University, 3-3-35, Yamate-cho, Suita, Osaka 564-8680, Japan

${ }^{2}$ Organization for Research and Development of Innovative Science and Technology, Kansai University, 3-3-35, Yamate-cho, Suita, Osaka 564-8680, Japan which consist of a densely cross-linked and brittle polyelectrolyte 1st network and a sparsely cross-linked and ductile neutral 2nd network, exhibit high mechanical strength (tensile fracture stress of $\sim 10 \mathrm{MPa}$ and tensile fracture strain of 1000-2000\%) and toughness (fracture energy of $\left.\sim 4000 \mathrm{~J} \mathrm{~m}^{-2}\right)^{13,14}$. The superior mechanical properties of DN hydrogels are attributed to energy dissipation through the internal fracture of the brittle 1st network. In addition, dynamic cross-links that reversibly dissociate and form in response to stimuli such as temperature, molecules, and stress are useful tools for designing not only responsive hydrogels but also tough hydrogels ${ }^{23-28}$. The abovementioned studies suggest that structural design is required to achieve effective energy dissipation in hydrogels.

During deformation, hydrogels exhibit viscoelastic behavior, which includes both viscous and elastic characteristics $^{29}$. While hydrogels are deformed by the application of stress, part of the total energy, which is based on loaded stress, is stored as elastic energy because of the elastic characteristic; however, the other part dissipates because of the viscous characteristic. The use of a large amount of chemical cross-linker results in the formation of rigid hydrogel networks in which the elastic characteristic contributes to their mechanical properties more predominantly 
than the viscous characteristic. In addition, we reported that hydrogels with a high cross-linker content have an inhomogeneous structure ${ }^{30}$, in which stress concentration within weak regions causes low fracture stress and strain. Therefore, to design tough hydrogels, we focused on the viscous characteristic to allow the applied stress to be relaxed by energy dissipation. A decrease in the cross-linker content enhances the contribution of the viscous characteristic to the mechanical properties of the hydrogels. Using our approach, it is possible to produce hydrogel networks with a high polymer chain density; the large molecular weight between cross-links enables effective energy dissipation by viscous characteristics because hydrogel networks have many entanglements that act as mobile cross-links. Herein, we report a simple and versatile method to produce tough and stretchable hydrogels by conventional radical polymerization without complicated structures or special preparation methods. Our strategy uses structural design to achieve effective energy dissipation. Specifically, the use of a high monomer concentration and low cross-linker content allows the formation of hydrogel networks with many entanglements, which produce physical cross-links (Fig. 1). In the obtained networks containing many entanglements, polymer chains can easily creep owing to the presence of minute amounts of chemical cross-links, enabling energy dissipation. To demonstrate that our strategy is universal, we used polyacrylamide (PAAm) and poly(2-methacryloyloxyethyl phosphorylcholine) (PMPC) as the main chains of the hydrogels, which have been widely used as representative hydrophilic polymers in various fields and biocompatible zwitterionic polymers in the biomedical field, respectively. While there have been studies on tough hydrogels that have been designed using unique cross-linkers ${ }^{9-11,17,18}$, this study demonstrates that tough and stretchable hydrogels can be prepared by conventional free radical polymerization without any special cross-linkers.

\section{Materials and methods \\ Materials}

Acrylamide (AAm), N, $N^{\prime}$-methylenebisacrylamide (MB AA), $N, N, N^{\prime}, N^{\prime}$-tetamethylethylenediamine (TEMED), and ammonium persulfate (APS) were purchased from Wako Pure Chemical Industries (Wako, Japan). 2-(Methacryloyloxy)ethyl phosphorylcholine (MPC) was supplied by NOF Corporation (Tokyo, Japan).

\section{Synthesis of poly(acrylamide) (PAAm) hydrogels}

AAm, which was recrystallized from benzene prior to use, MBAA as a cross-linker, and TEMED were dissolved in deionized water to prepare a monomer solution with a total volume of $2.42 \mathrm{~mL}$. In deionized water, APS, as an initiator, was also dissolved. The aqueous monomer and APS solutions were degassed using freeze-pump-thaw cycles and purged with Ar. After the freeze-pump-thaw cycles, the aqueous APS solution $(0.08 \mathrm{~mL})$ was added to the monomer solution in an ice bath to prevent premature gelation. The resulting mixture containing APS and TEMED in concentrations of $0.47 \times 10^{-3} \mathrm{~mol} / \mathrm{L}$ and $4.0 \times 10^{-3} \mathrm{~mol} / \mathrm{L}$, respectively, was poured into a mold composed of two slides separated with a 5-mm-thick glass spacer (for compression tests) or a 1.76-mm-thick glass spacer (for tensile tests) (Fig. S1). The copolymerization of AAm and MBAA was performed at $25^{\circ} \mathrm{C}$ for 1 day. Asprepared PAAm hydrogels were obtained by removing the molds. Swollen PAAm hydrogels were prepared by immersing the as-prepared hydrogels in water until their swelling ratio reached equilibrium.

\section{Synthesis of poly(2-methacryloyloxyethyl phosphorylcholine) (PMPC) hydrogels}

MPC as the main monomer, MBAA as a cross-linker, and TEMED were dissolved in deionized water to prepare a monomer solution with a total volume of $0.92 \mathrm{~mL}$. APS, as an initiator, was dissolved in deionized water to prepare an initiator solution. The aqueous monomer and initiator solutions were degassed using six freeze-pump-thaw cycles and purged with Ar. After the freeze-pump-thaw cycles, the aqueous APS solution $(0.08 \mathrm{~mL})$ was added to the monomer solution in an ice bath to prevent premature gelation. The resulting mixture of APS and TEMED at concentrations of $0.47 \times 10^{-3} \mathrm{~mol} / \mathrm{L}$ and $4.0 \times 10^{-3} \mathrm{~mol} / \mathrm{L}$, respectively, was poured into a mold composed of two slides separated with a 5-mm-thick glass spacer (for compression tests) or a 1.76-mm-thick glass spacer (for tensile tests) (Fig. S1); copolymerization of MPC and MBAA was performed at $25^{\circ} \mathrm{C}$ for 1 day. As-prepared PMPC hydrogels were obtained by removing the molds.

\section{Conversion measurements}

The as-prepared PAAm hydrogels were washed by immersion in deionized water for 2 weeks to completely remove unreacted monomers and initiators. Then, after the PAAm gels were dried at $70^{\circ} \mathrm{C}$ in an oven for 2 days, their weight $\left(W_{\text {dried gel }}\right)$ was measured. $W_{\text {dried gel }}$ represents the total weight of polymerized AAm and MBAA in the resulting PAAm networks. The conversion of the monomers after gel formation was determined from the $W_{\text {dried gel }}$ and the total weight ( $\left.W_{\text {monomer }}\right)$ of AAm and MBAA in a feed solution using Eq. (1).

$$
\text { Conversion }(\%)=\frac{W_{\text {driegel }}}{W_{\text {monomer }}} \times 100
$$

\section{Measurement of the water content of the hydrogels}

PAAm hydrogels were immersed in deionized water until their swelling ratio reached equilibrium. Then, the 
a
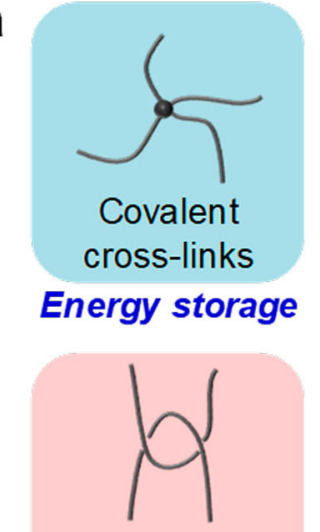

Entanglement cross-links

Energy dissipation

b

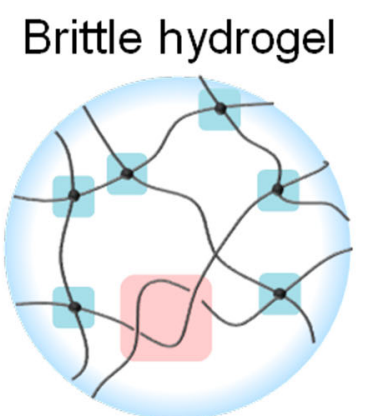

Many chemical cross-links Few physical cross-links

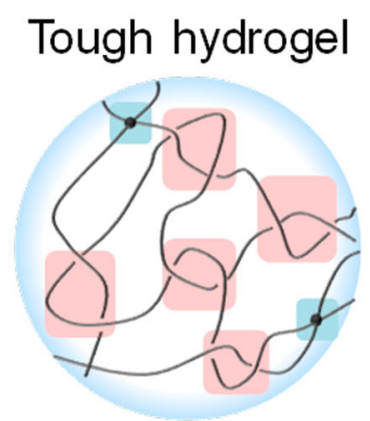

Few chemical cross-links Many physical cross-links

C

Brittle hydrogel

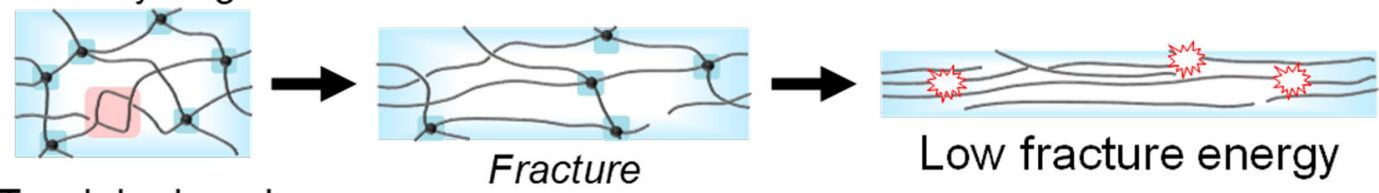

Tough hydrogel
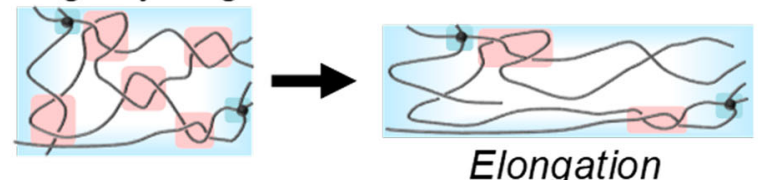
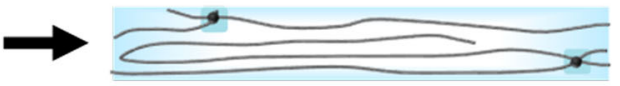

High fracture energy

Fig. 1 Schematics of hydrogels with physical and chemical cross-links. a Covalent and entanglement cross-links for energy storage and dissipation, respectively. $\mathbf{b}$ Chemically and physically cross-linked structures of brittle and tough hydrogels. $\mathbf{c}$ Fracture behavior of brittle and tough hydrogels with few and many entanglements, respectively.

weight ( $\left.W_{\text {swollen gel }}\right)$ of the swollen hydrogels was measured. After they were dried at $70{ }^{\circ} \mathrm{C}$ in an oven for 2 days, the weight $\left(W_{\text {dried gel }}\right)$ of the resulting dried gels was measured. The equilibrium water content of the PAAm hydrogels was determined using Eq. (2).

$$
\text { Water content }(\%)=\frac{W_{\text {swollengel }}-W_{\text {dried gel }}}{W_{\text {swollen gel }}} \times 100
$$

\section{Mechanical tests}

Compression tests of the as-prepared and swollen PAAm and PMPC hydrogels, which were prepared using the molds shown in Fig. S1, were performed using a mechanical testing instrument (SMT1-2-N, Shimadzu Co. Ltd., Kyoto) with a compression velocity of $10 \mathrm{~mm} / \mathrm{min}^{-1}$. Tensile tests of the as-prepared and swollen PAAm and PMPC hydrogels were performed using a mechanical testing instrument with crosshead speeds of 5,50 , and $500 \mathrm{~mm} / \mathrm{min}^{-1}$. The toughness of the hydrogels, which is the work to fracture, was determined from the area under the tensile stress-strain curve of an unnotched sample with a length of $20 \mathrm{~mm}$.

\section{Determination of the cross-linking density of the hydrogels}

The elastic modulus of the hydrogels was determined from the strain-stress curves obtained by the compression tests using Eq. (3).

$$
\sigma=G\left(\alpha-\alpha^{-2}\right)
$$

where $\sigma$ is the compression stress, $G$ is the elastic modulus, and $\alpha$ is the ratio of the thickness of the gel before and after compression. The plot of $\sigma$ vs. $\left(\alpha-\alpha^{-2}\right)$ showed a linear relationship. The slope of this line provided the $G$ value. The effective cross-linking density, 
$v_{\mathrm{e}}$, of the gel was determined by Eq. (4).

$$
G \approx R T \nu_{e} \phi^{1 / 3}
$$

\section{Dynamic mechanical analysis}

Dynamic mechanical analysis of the hydrogels was conducted using a nonresonance forced vibration viscoelastometer (Rheogel-E-4000F; UBM, Kyoto, Japan) in tension mode. The frequency and amplitude of the vibration were adjusted to $100 \mathrm{~Hz}$ and $50 \mu \mathrm{m}$, respectively. From the dynamic mechanical analysis, we determined the storage modulus $\left(G^{\prime}\right)$, loss modulus $\left(G^{\prime \prime}\right)$ and loss factor $\left(\tan \delta=G^{\prime \prime} / G^{\prime}\right)$ to evaluate the viscoelastic properties of the hydrogels prepared under various conditions.

\section{Results and discussion}

\section{Mechanical properties of PAAm hydrogels}

First, to design polymer networks with many entanglements and a minute number of chemical cross-links, we prepared PAAm hydrogels, which are the most standard hydrogels, by the copolymerization of AAm as the main monomer and MBAA as a standard cross-linker using a wide range of monomer concentrations and cross-linker contents. The mechanical properties of the as-prepared PAAm hydrogels synthesized with various monomer concentrations and cross-linker contents were evaluated by compression and tensile tests (Fig. 2). Polymerization with high monomer concentrations $(2.5 \mathrm{~mol} / \mathrm{L}$ and $5.0 \mathrm{~mol} / \mathrm{L}$ ) allowed self-standing PAAm hydrogels with a cross-linker content of $0.001 \mathrm{~mol} \%$ to form despite the low cross-linker content. As-prepared PAAm hydrogels with a cross-linker content greater than $0.1 \mathrm{~mol} \%$ easily broke during compression (Fig. 2a-d and Movie S1b). However, the as-prepared PAAm hydrogels with a crosslinker content lower than $0.1 \mathrm{~mol} \%$ did not break at up to 95\% strain and 6-MPa stress (Fig. 2c and Movie S1a). From the work of Sakai et al. ${ }^{31}$, the stress-strain curve of a four-arm poly(ethylene glycol) (tetra-PEG) hydrogel is replotted in Fig. 2a-c. The as-prepared tetra-PEG hydrogel is tough owing to its homogeneous network structure. Notably, although standard free radical polymerization results in the formation of an inhomogeneous network structure ${ }^{30}$, the as-prepared PAAm hydrogels prepared under polymerization conditions with a high monomer concentration and a low cross-linker content were tougher than the tetra-PEG hydrogel with a homogeneous network structure.

In the tensile tests, the as-prepared PAAm hydrogels with a high cross-linker content of more than $0.1 \mathrm{~mol} \%$ could slightly extend, while the hydrogels prepared with a $2.5 \mathrm{~mol} / \mathrm{L}$ monomer concentration and less than $0.01 \mathrm{~mol}$ $\%$ cross-linker content could be elongated by more than ten times and exhibited the highest fracture strain
(Fig. 2e-g, i and Movie S2). The stress-strain curve of the hydrogel with a cross-linker content greater than $0.5 \mathrm{~mol}$ $\%$ is a straight line. Conventional polymer materials, such as elastomers, demonstrate $\mathrm{S}$-shaped stress-strain curves because polymer chains result in extension from a threadlike shape. These results show that a large number of chemical cross-links cause brittleness of the PAAm hydrogels because the extension of the polymer chains is inhibited, and the stress is concentrated in weak chains within inhomogeneous networks. However, the fracture stress of the PAAm hydrogels was effectively improved with an increase in the monomer concentration during polymerization. The fracture stress of the PAAm hydrogels prepared with a $5.0 \mathrm{~mol} / \mathrm{L}$ monomer concentration and $0.005 \mathrm{~mol} \%$ cross-linker content was approximately $300 \mathrm{kPa}$. At the same cross-linker content, the initial slopes of the stress-strain curves of hydrogels synthesized with a $5.0 \mathrm{~mol} / \mathrm{L}$ monomer concentration were steeper than those of hydrogels synthesized with a $2.5 \mathrm{~mol} / \mathrm{L}$ monomer concentration. The steeper initial slopes indicate that the former hydrogels had a higher elastic modulus than the latter despite the same cross-linker content. For example, even though PAAm hydrogels were prepared using the same cross-linker content of $5.0 \mathrm{~mol} \%$, the Young's moduli of the hydrogels prepared with AAm concentrations of $1.0,2.5$, and $5.0 \mathrm{~mol} / \mathrm{L}$ were determined to be 9.6, 40.0, and $196.7 \mathrm{kPa}$ from the initial slopes of the stress-strain curves. These results suggest that the high monomer concentration during polymerization causes physical chain entanglements that act as mobile crosslinks. Therefore, the improved fracture stress is attributed to the high polymer chain density in the hydrogel formed at a high monomer concentration. Of note, when only AAm was polymerized at a monomer concentration of $5.0 \mathrm{~mol} / \mathrm{L}$, a self-standing PAAm hydrogel formed despite the absence of a chemical cross-linker. Many polymer chain entanglements, which result from polymerization with a high monomer concentration, act as physical crosslinks. However, when the strain of the PAAm hydrogel without a chemical cross-linker was greater than 2, the stress was quickly relaxed by creeping of the polymer chains, and the hydrogel easily broke upon application of low stress. These results suggest that the conditions for preparing tough and stretchable hydrogels require polymerization with a high monomer concentration and a minute amount of cross-linker.

Some papers have reported that as-prepared hydrogels designed using unique strategies exhibit high toughness; however, most of them do not exhibit the mechanical properties of swollen hydrogels after equilibrium swelling in aqueous media. In addition, the mechanical properties of swollen hydrogels are important because these hydrogels are typically utilized in aqueous media. Compression and tensile tests of swollen PAAm hydrogels demonstrated that 


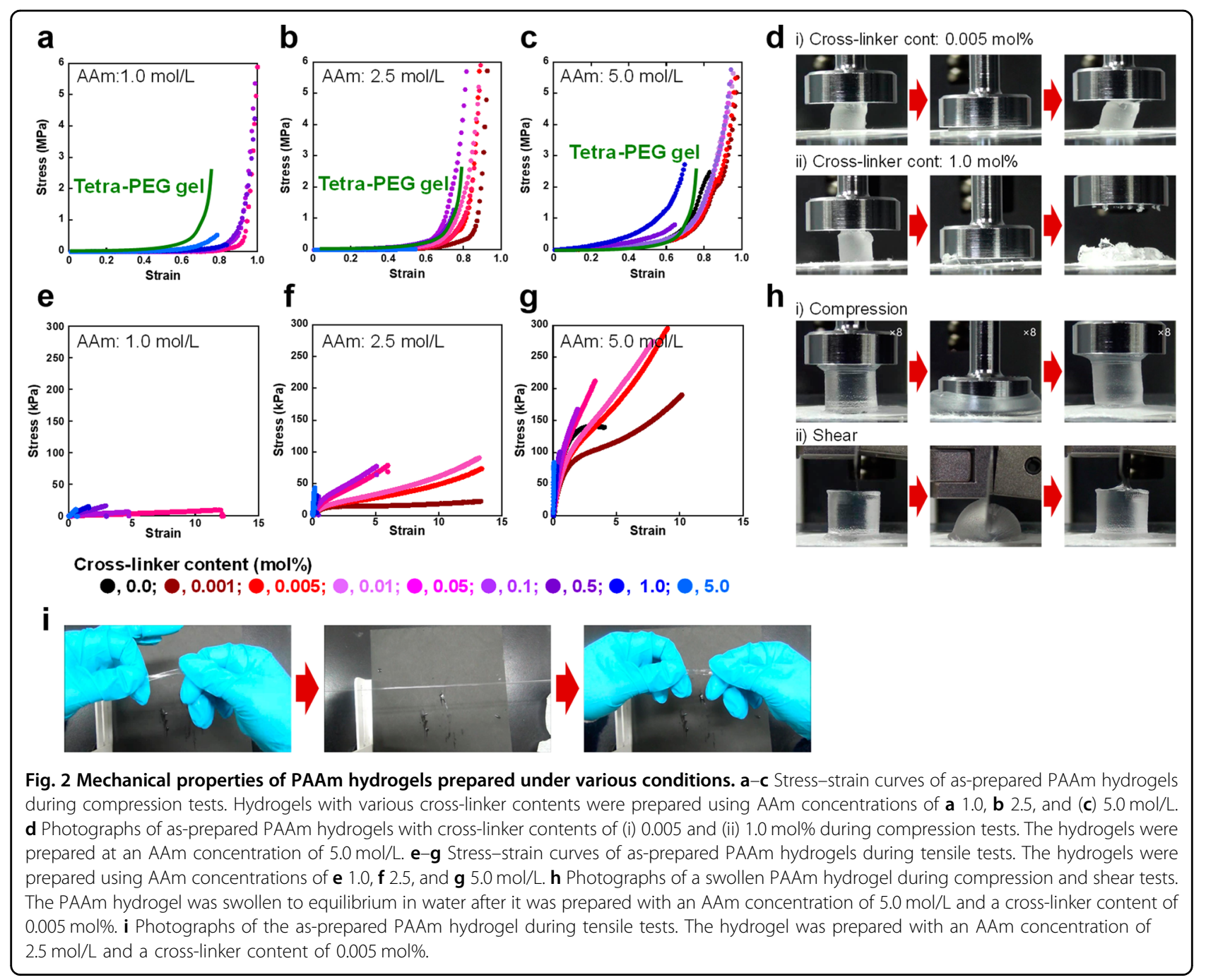

their fracture strain and stress decreased after equilibrium swelling in aqueous media. The water content of the swollen PAAm hydrogels after equilibrium swelling decreased with increasing cross-linker content (Fig. S2). It should be noted that the swollen PAAm hydrogels prepared with a higher AAm concentration had a lower water content than those prepared with a lower AAm concentration even though they were prepared with the same cross-linker content. This means that polymerization with high monomer concentrations is likely to induce polymer chain entanglements in the resulting hydrogel networks. The decreased fracture stress and strain of swollen hydrogels are caused by an increase in the water content (Fig. S3). Because polymer chains are expanded in swollen hydrogels, they cannot be expanded further by applying stress. In addition, entanglements as physical cross-links are partially loosened during the swelling of hydrogels in aqueous media. Thus, swollen hydrogels break under a smaller strain than the as-prepared hydrogels because the applied stress is not effectively dissipated. However, the swollen PAAm hydrogels prepared under polymerization conditions of a high monomer concentration and low cross-linker content retain their high toughness despite equilibrium swelling (Figs. S2 and S3). For the swollen hydrogels with a $0.005 \mathrm{~mol} \%$ cross-linker content, even if a large strain of more than $90 \%$ is applied during compression tests or a large elongation of more than seven times is applied during tensile tests, these hydrogels do not fracture despite the high water content of more than $90 \%$, and they recover to their original shape after the stress is released (Fig. 2h, Figs. S2, S3 and Movie S3a). Of note, the swollen hydrogels prepared with a monomer concentration of $5.0 \mathrm{~mol} \%$ and a cross-linker content of $0.005 \mathrm{~mol} \%$ cannot be cut with a knife despite their swollen state (Fig. $2 \mathrm{~h}$ and Movie S3b). Thus, the as-prepared and swollen PAAm hydrogels prepared with a high monomer concentration and a low cross-linker content demonstrate high mechanical toughness and high stretchability. 

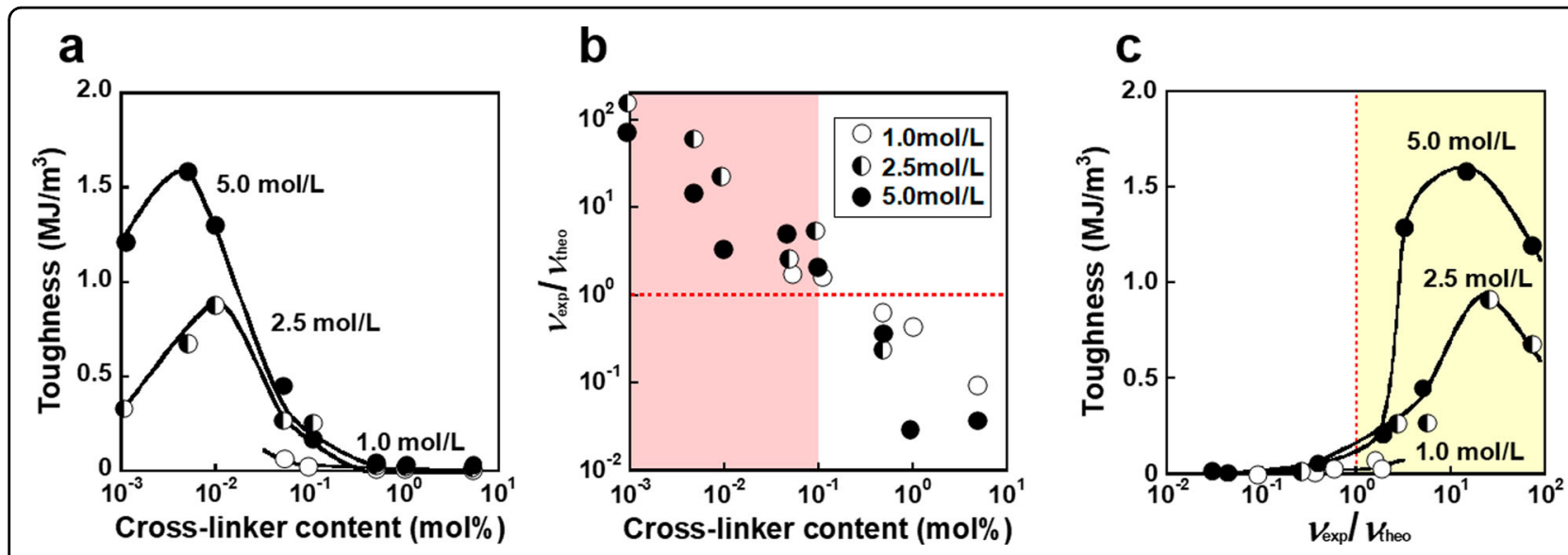

Fig. 3 Toughness of PAAm hydrogels with various cross-linked structures. a Relationship between the cross-linker content and toughness of asprepared PAAm gels prepared under various preparation conditions. The gels were prepared at AAm concentrations of $1.0(\mathrm{O}), 2.5(\mathbf{O})$, and $5.0 \mathrm{~mol} / \mathrm{L}$ (O). b Effect of the cross-linker content on the cross-linking ratio between the experimental and theoretical cross-linking densities $\left(V_{\text {exp }} / V_{\text {theo }}\right)$ of the PAAm gels prepared under various conditions. c Relationship between $v_{\text {exp }} / v_{\text {theo }}$ and toughness of the PAAm gels prepared under various conditions.

\section{Relationship between the cross-linked structure and toughness of PAAm hydrogels}

To elucidate the mechanism by which hydrogels become tough and stretchable, we determined the toughness of PAAm hydrogels from the stress-strain curve during tensile tests. In general, the fracture energies of polymeric materials are determined from the stress-strain curve of notched samples ${ }^{6,32,33}$. However, we were not able to notch the PAAm hydrogels synthesized in this study because they were very tough. In this study, we defined toughness from the area under the tensile stress-strain curve of an unnotched sample. The toughness is larger than the fracture energy determined using a notched sample because it includes energies not only for growing cracks but also for notching. The PAAm hydrogels prepared with a cross-linker content of more than $0.1 \mathrm{~mol} \%$ have a much lower toughness than those prepared with a cross-linker content less than $0.1 \mathrm{~mol} \%$ (Fig. 3a). An increase in the monomer content during the polymerization considerably enhanced the toughness of the resulting hydrogels. Of note, the PAAm hydrogel prepared with a monomer concentration of $5.0 \mathrm{~mol} / \mathrm{L}$ and a cross-linker concentration of $0.005 \mathrm{~mol} \%$ exhibited the maximum toughness of $1.6 \mathrm{MJ} / \mathrm{m}^{3}$, although the toughness could not be directly compared with the fracture energy of tough hydrogels prepared by different strategies $^{13,15,33,34}$.

In general, the experimental cross-linking density ( $\left.v_{\text {exp }}\right)$ of hydrogels can be determined from their elastic modulus. For example, the $v_{\exp }$ of the as-prepared PAAm hydrogels prepared with an AAm concentration of $1.0 \mathrm{~mol} / \mathrm{L}$ increased gradually with an increase in the cross-linker content (Fig. S4). Although it is natural that an increase in the cross-linker content results in increasing $v_{\text {exp }}$ of the resulting PAAm hydrogels, not only chemical cross-links based on MBAA but also physical cross-links based on entanglements are included in the $v_{\text {exp }}$ determined from the elastic modulus. To evaluate the chemical and physical cross-links based on MBAA and entanglements, respectively, we determined the theoretical cross-linking density $\left(v_{\text {theo }}\right)^{35}$ and $v_{\exp }$ of the PAAm hydrogels prepared under various conditions. If the ratio of experimental to theoretical cross-linking densities $\left(v_{\text {exp }} / v_{\text {theo }}\right)$ is less than one, unreacted polymerizable groups of MBAA remain in the PAAm hydrogels, and not all MBAA is involved in chemical cross-links. The PAAm hydrogels with $v_{\text {exp }} / v_{\text {theo }}$ ratios greater than one contained physical cross-links that were based on polymer chain entanglements in addition to chemical cross-links based on MBAA. The $v_{\text {exp }} / v_{\text {theo }}$ ratio of the PAAm hydrogels monotonically decreased with an increase in the crosslinker content (Fig. 3b). The PAAm hydrogel prepared with a cross-linker content of approximately $0.1 \mathrm{~mol} \%$ has a $v_{\text {exp }} / v_{\text {theo }}$ ratio of one, which means that the crosslinking density determined from the elastic modulus is equal to that based on a chemical cross-linker. Specifically, the PAAm hydrogels prepared with a cross-linker content of less than $0.1 \mathrm{~mol} \%$ have physical cross-links that are based on entanglements because they have $v_{\exp } /$ $v_{\text {theo }}$ ratios greater than one, while the PAAm hydrogels with $v_{\text {exp }} / v_{\text {theo }}$ ratios less than one have a very low toughness, and the toughness increases considerably with an increase in $v_{\text {exp }} / v_{\text {theo }}$ to greater than one (Fig. 3c). These results suggest that the entanglement of polymer chains contributes to the high toughness of the PAAm hydrogel with a monomer concentration of $5.0 \mathrm{~mol} / \mathrm{L}$ and a cross-linker content of $0.005 \mathrm{~mol} \%$. In polymer networks with many entanglements, because the applied 

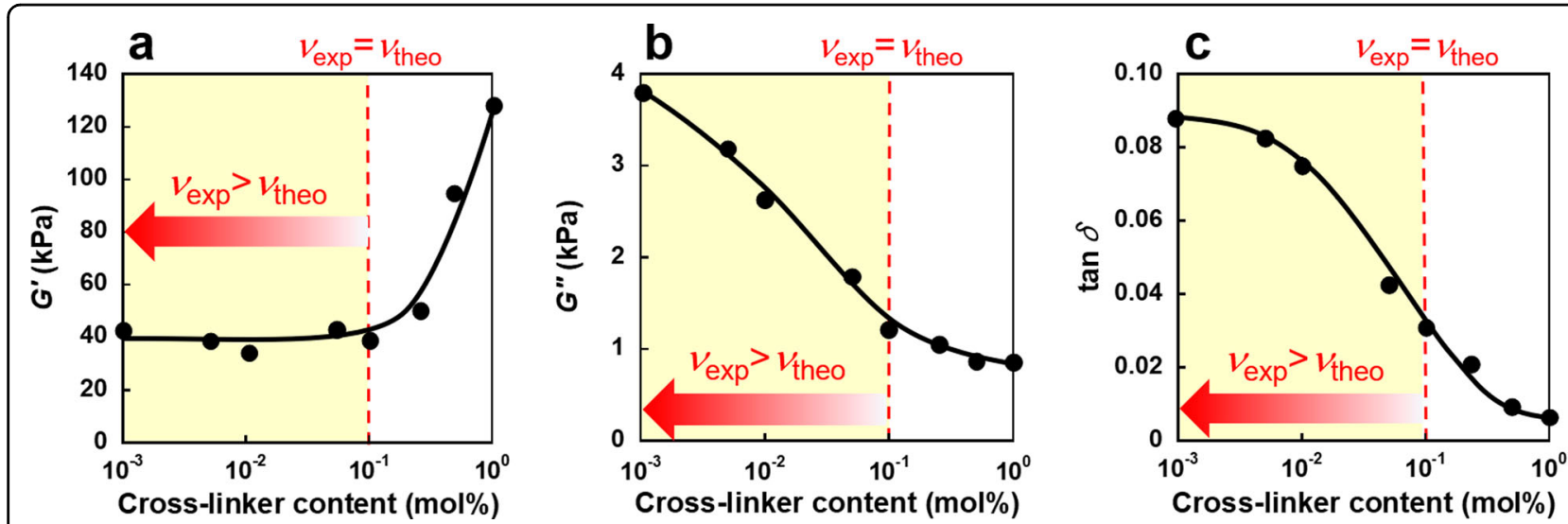

Fig. 4 Dynamic mechanical properties of PAAm hydrogels with various cross-linked structures. Effect of the cross-linker content on the storage modulus $\left(G^{\prime}\right)(\mathbf{a})$, loss modulus $\left(G^{\prime \prime}\right)(\mathbf{b})$, and loss factor $(\tan \delta)(\mathbf{c})$ of the as-prepared PAAm hydrogels prepared at an AAm concentration of $2.5 \mathrm{~mol} / \mathrm{L}$ and various cross-liner contents.

stress is dissipated by creeping of the polymer chains entangled in the networks, the hydrogels become tough and stretchable. In fact, we observed creeping of the hydrogel prepared with AAm concentrations of 5.0 and $10 \mathrm{~mol} / \mathrm{L}$ after they were elongated up to strains of 7 and 4 , respectively (Fig. S5).

Our strategy for preparing tough hydrogels uses the viscous characteristic to allow the applied stress to be relaxed by energy dissipation. In this strategy, a decrease in the cross-linker content enhances the contribution of the viscous characteristic to the mechanical properties of the hydrogels. Dynamic mechanical analysis is useful for evaluating the contribution of the elastic and viscous characteristics to the mechanical properties of hydrogels. We carried out dynamic mechanical analysis of PAAm hydrogels prepared with an AAm concentration of $2.5 \mathrm{~mol} / \mathrm{L}$ and various cross-linker contents. When the cross-linker content of the PAAm hydrogels decreased, their storage modulus $\left(G^{\prime}\right)$ decreased, and the loss modulus $\left(G^{\prime \prime}\right)$ increased (Fig. 4). As a result, the loss factor $\tan \delta$, which is the ratio of $G^{\prime \prime}$ to $G^{\prime}$, of the PAAm hydrogels increased substantially with a decrease in the cross-linker content. In particular, the PAAm hydrogel with a cross-linker content of $0.005 \mathrm{~mol} \%$ had an approximately twelve times greater $\tan \delta$ than that with a cross-linker content of $1 \mathrm{~mol} \%$. As shown in Fig. 3b, the $v_{\text {exp }} / v_{\text {theo }}$ ratio of the PAAm hydrogels with a cross-linker content of less than $0.1 \mathrm{~mol} \%$ was greater than one, revealing that the entanglements of the polymer chains increased in the hydrogel networks. Therefore, an increase in $\tan \delta$ coincides with an increase in $v_{\exp } / v_{\text {theo. }}$. In addition, the constant $G^{\prime}$ in the regime of $v_{\exp }>v_{\text {theo }}$, as shown in Fig. 4a, indicates that the storage modulus of the PAAm hydrogels is determined by the density of the trapped entanglements. This implies that the PAAm hydrogels in the regime of $v_{\exp }>v_{\text {theo }}$ were mainly cross- linked by the polymer chain entanglement. Although Fig. 4 might not be strong evidence, we think that an increase in $G^{\prime \prime}$ plays an important role in making hydrogels tough despite the slight increase in $\tan \delta$. From these results, we propose a possible mechanism by which the polymer chain entanglements enhance the contribution of the viscous characteristic, which enables energy dissipation, to the mechanical properties of the hydrogels. As a result, tough and stretchable hydrogels can be easily prepared by tuning the conditions for the preparation of networks with many entanglements. Further works about dynamic mechanical analysis and homogeneity of the networks will be performed to clarify the detailed mechanism.

\section{Tough and stretchable zwitterionic polymer hydrogels}

To demonstrate that the use of a high monomer concentration and low cross-linker content is a universal method for easily making hydrogels tough and stretchable, we used 2-methacryloyloxyethyl phosphorylcholine (MPC), which has been widely used as a biocompatible zwitterionic polymer in the biomedical field, instead of AAm to prepare hydrogels. In general, MPC has been widely utilized to prepare biocompatible polymers. Although hydrogels based on MPC have many potential applications in contact lenses, artificial joints, and other biomaterials ${ }^{36}$, they have a considerable disadvantage of low mechanical strength (i.e., they are brittle and weak). We tried to prepare tough and stretchable hydrogels using MPC by only tuning the preparation conditions of high monomer concentration and low cross-linker content. To optimize the conditions to prepare hydrogel networks with many entanglements, poly(2-methacryloyloxyethyl phosphorylcholine) (PMPC) hydrogels were prepared by the copolymerization of MPC and MBAA with a wide range of monomer concentrations and cross-linker contents. Similar to PAAm hydrogels, the PMPC hydrogels 

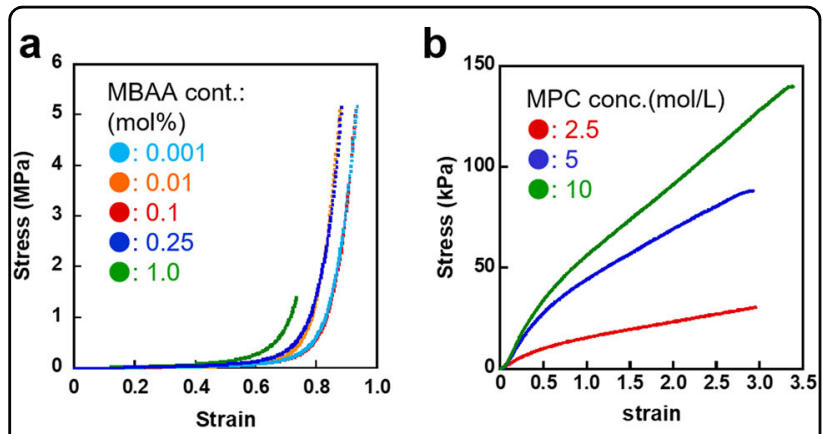

C i) Cross-linker cont: $0.1 \mathrm{~mol} \%$

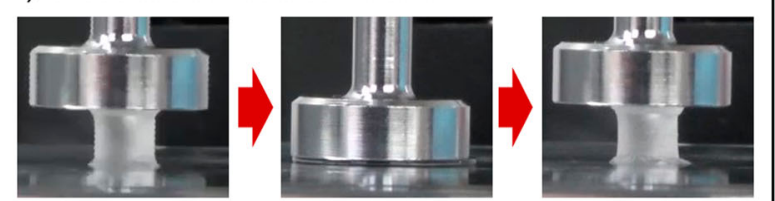

ii) Cross-linker cont: $1.0 \mathrm{~mol} \%$

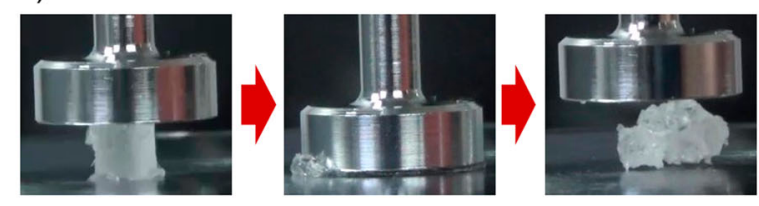

d i) Cross-linker cont: $0.1 \mathrm{~mol} \%$

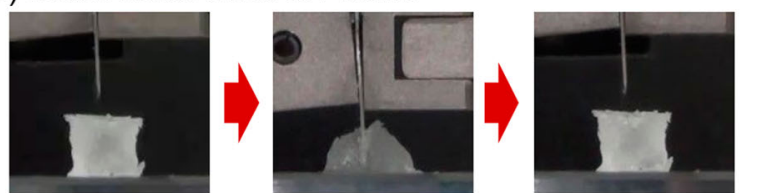

ii) Cross-linker cont: $1.0 \mathrm{~mol} \%$

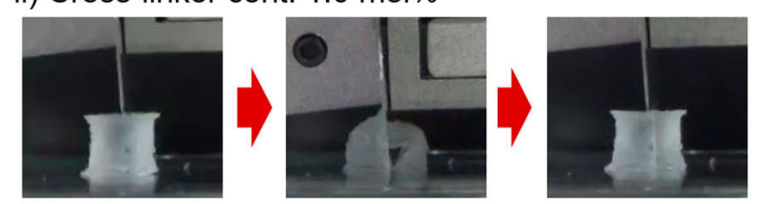

e

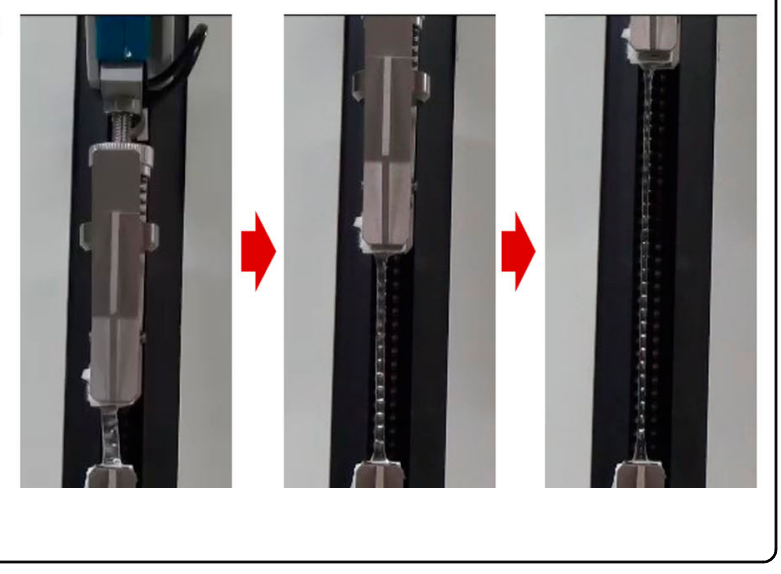

with a high cross-linker content easily broke upon application of a low stress in compression tests; however, the PMPC hydrogels with a cross-linker content of less than $0.1 \mathrm{~mol} \%$ did not break at up to a $95 \%$ strain and 6 -
Fig. 5 Mechanical properties of PMPC hydrogels prepared under various conditions. a Stress-strain curves of as-prepared PMPC hydrogels with various cross-linker contents during compression tests. The hydrogels were prepared using an MPC concentration of $5.0 \mathrm{~mol} /$ L. b Stress-strain curves of as-prepared PMPC hydrogels with MPC concentrations of $2.5,5$, and $10 \mathrm{~mol} / \mathrm{L}$ during tensile tests. The hydrogels were prepared using a cross-linker content of $0.1 \mathrm{~mol} \%$. c Photographs of as-prepared PMPC hydrogels with cross-linker contents of (i) 0.1 and (ii) 1.0 mol\% during compression tests. The hydrogels were prepared at an MPC concentration of $2.5 \mathrm{~mol} / \mathrm{L}$. d Photographs of as-prepared PMPC hydrogels with cross-linker contents of (i) 0.1 and (ii) 1.0 mol\% during shear tests. The hydrogels were prepared at an MPC concentration of $2.5 \mathrm{~mol} / \mathrm{L}$. e Photographs of the as-prepared PMPC hydrogel during tensile tests. The hydrogel was prepared under an MPC concentration of $5.0 \mathrm{~mol} / \mathrm{L}$ and a crosslinker content of $0.1 \mathrm{~mol} \%$.

MPa stress (Fig. 5a, c and Movie S4). Of note, the PMPC hydrogel with a cross-linker content of $0.1 \mathrm{~mol} \%$ could not be cut with a knife (Fig. 5d and Movie S5). In tensile tests, the PMPC hydrogels with many entanglements underwent large elongations and exhibited the highest fracture strain, differing from the general MPC-based hydrogels (Fig. 5b, e and Movie S6). The Young's moduli of the PMPC hydrogels prepared with MPC concentrations of $2.5,5$, and $10 \mathrm{~mol} / \mathrm{L}$ and with the same crosslinker content of $0.1 \mathrm{~mol} \%$ were determined to be 16.6 , 60.4 , and $74.0 \mathrm{kPa}$ from the initial slopes of the stress-strain curves, respectively. It should be noted that the PMPC hydrogel prepared with a higher MPC concentration exhibited a greater Young's modulus than that prepared with a lower MPC concentration even though the PMPC hydrogels were prepared using the same crosslinker content. Similar to the formation of the PAAm networks, the preparation conditions with a high monomer concentration and a low cross-linker content resulted in the formation of networks with many entanglements of PMPC chains rather than chemical cross-links based on MBAA. Therefore, we conclude that preparation with a high monomer concentration and low cross-linker content is a universal method for preparing tough and stretchable hydrogels because the resulting networks have many physical cross-links based on polymer chain entanglements that act as mobile cross-links for energy dissipation. Thus, tough and stretchable hydrogels can be easily prepared by optimizing the preparation conditions to form many polymer chain entanglements without using complicated methods.

\section{Conclusion}

In summary, we have demonstrated a simple and versatile strategy for producing tough and stretchable hydrogels by free radical polymerization of standard hydrophilic monomers. Our strategy is to only tune the polymerization conditions without introducing a special structure or using 
complicated methods; we can optimize the network structures, which have many polymer chain entanglements for energy dissipation by polymerization conditions with a high monomer concentration and a low cross-linker content. The hydrogels prepared under the optimized conditions have a $v_{\text {exp }} / v_{\text {theo }}$ ratio greater than one, indicating that the hydrogels contain physical cross-links based on polymer chain entanglements in addition to chemical cross-links based on MBAA. The toughness of the hydrogels increased considerably with an increase in $v_{\text {exp }} / v_{\text {theo }}$ above than one. Although our strategy uses neither a special structure nor a complicated method, the hydrogels prepared using our strategy exhibited high toughness. Tough and stretchable nonionic PAAm and zwitterionic PMPC prepared under optimized polymerization conditions undergo large elongations, exhibit high fracture strain and cannot be cut with a knife because of the many entanglements as physical crosslinks. Our strategy is applicable to preparing tough and stretchable hydrogels from a variety of polymers. Structural design using polymer chain entanglements for energy dissipation to overcome the limitation of low mechanical performance will lead to many practical uses of hydrogels.

\section{Acknowledgements}

This work was supported in part by a Grant-in-Aid for Scientific Research (B) (No. JP2OH04539) from the Japan Society of the Promotion of Science (JSPS); by a Grant-in-Aid for Scientific Research on Innovative Areas of "Aquatic Functional Materials" (No. JP2OH05236) and the Private University Research Branding Project: Matching Fund Subsidy from Ministry of Education, Culture, Sports, Science and Technology (MEXT) Japan (2016-2020); by the AMED Sinnovation Program for the development of biofunctional materials for the realization of innovative medicine; and by research grants from the Canon Foundation.

\section{Author contributions}

T.M. conceived the idea, designed the experiments, and supervised the project. C.N., Y.I, and C.H. performed experiments. All authors discussed the results and contributed to the data interpretation. C.N. and T.M. wrote the manuscript. T.M. edited and revised the manuscript.

\section{Conflict of interest}

The authors declare no competing interests.

\section{Publisher's note}

Springer Nature remains neutral with regard to jurisdictional claims in published maps and institutional affiliations.

Supplementary information The online version contains supplementary material available at https://doi.org/10.1038/s41427-021-00302-2.

Received: 10 January 2021 Revised: 22 February 2021 Accepted: 1 March 2021.

Published online: 9 April 2021

\section{References}

1. Hoffman, A. S. Hydrogels for biomedical applications. Adv. Drug Deliv. Rev. 54, 3-12 (2002).

2. Peppas, N. A., Hilt, J. Z., Khademhosseini, A. \& Langer, R. Hydrogels in biology and medicine: from molecular principles to bionanotechnology. Adv. Mater. 18, 1345-1360 (2006).
3. Li, J. \& Mooney, D. J. Designing hydrogels for controlled drug delivery. Nat. Rev. Mater. 1, 1-17 (2016).

4. Zhang, Y. S. \& Khademhosseini, A. Advances in engineering hydrogels. Science 356, eaaf3627 (10pp) (2017)

5. Buwalda, S. J., Vermonden, T. \& Hennink, W. E. Hydrogels for therapeutic delivery: current developments and future directions. Biomacromolecules 18, 316-330 (2017).

6. Lake, G. J. \& Thomas, A. G. The strength of highly elastic materials. Proc. R. Soc. A 300, 108-119 (1967).

7. Lake, G. J. Fatigue and fracture of elastomers. Rubber Chem. Technol. 68 435-460 (1995).

8. Simha, N. K., Carlson, C. S. \& Lewis, J. L. Evaluation of fracture toughness of cartilage by micropenetration. J. Mater. Sci. Mater. Med. 14, 631-639 (2003).

9. Okumura, Y. \& Ito, K. The polyrotaxane gel: a topological gel by figure-of-eight cross-links. Adv. Mater. 13, 485-487 (2001).

10. Noda, Y., Hayashi, Y. \& Ito, K. From topological gels to slide-ring materials. J. Appl. Polym. Sci. 131, 40509 (2014). (9pp).

11. Haraguchi, K. \& Takehisa, T. Nanocomposite hydrogels: a unique organicinorganic network structure with extraordinary mechanical, optical, and swelling/de-swelling properties. Adv. Mater. 14, 1120-1124 (2002).

12. Gong, J. P., Katsuyama, Y., Kurokawa, T. \& Osada, Y. Double-network hydrogels with extremely high mechanical strength. Adv. Mater. 15, 1155-1158 (2003).

13. Gong, J. P. Why are double network hydrogels so tough? Soft Matter $\mathbf{6}$, 2583-2590 (2010).

14. Nakaijma, T. Generalization of the sacrificial bond principle for gel and elastomer toughening. Polym. J. 49, 447-485 (2017).

15. Sun, J.-Y. et al. Highly stretchable and tough hydrogels. Nature 489, 133-136 (2012).

16. Ducrot, E., Chen, Y., Bulters, M., Sijbesma, R. P. \& Creton, C. Toughening elastomers with sacrificial bonds and watching them break. Science $\mathbf{3 4 4}$, 186-189 (2014).

17. Imran, A. B. et al. Extremely stretchable thermosensitive hydrogels by introducing slide-ring polyrotaxane cross-linkers and ionic groups into the polymer network. Nat. Commun. 5, 5124 (2014). (8pp).

18. Sun, G., Li, Z., Liang, R., Weng, L.T. \& Zhang, L. Super stretchable hydrogel achieved by non-aggregated spherulites with diameters $<5 \mathrm{~nm}$. Nat. Commun. 7, 12095 (2016). (8pp).

19. Filippidi, E. et al. Toughening elastomers using mussel-inspired iron-catechol complexes. Science 358, 502-505 (2017).

20. Gotoh, H. et al. Optically transparent, high-toughness elastomer using a polyrotaxane cross-linker as a molecular pulley. Sci. Adv. 4, eaat7629 (2018).

21. Ke, H. et al. Shear-induced assembly of a transient yet highly stretchable hydrogel based on pseudopolyrotaxanes. Nat. Chem. 11, 470-477 (2019).

22. Matsuda, T., Kawakami, R., Namba, R., Nakajima, T. \& Gong, J. P. Mechanoresponsive self-growing hydrogels inspired by muscle training. Science $\mathbf{3 6 3}$, 504-508 (2019).

23. Sinawang, G., Osaki, M., Takashima, Y., Yamaguchi, H. \& Harada, A. Biofunctional hydrogels based on host-guest interactions. Polym. J. 52, 839-859 (2020).

24. Nakahata, M., Takashima, Y. \& Harada, A. Highly flexible, tough, and self-Healing supramolecular polymeric materials using host-guest interaction. Macromol. Rapid Commun. 37, 86-92 (2016).

25. Miyata, T. Preparation of smart soft materials using molecular complexes. Polym. J. 42, 277-289 (2010).

26. Miyata, T., Asami, N. \& Uragami, T. A reversibly antigen-responsive hydrogel. Nature 399, 766-769 (1999).

27. Miyata, T., Jige, M., Nakaminami, T. \& Uragami, T. Tumor marker-responsive behavior of gels prepared by biomolecular imprinting. PNAS 103, 1190-1193 (2006).

28. Matsumoto, K. Sakikawa, N. \& Miyata, T. Thermo-responsive gels that absorb moisture and ooze water. Nat. Commun. 9, 2315 (2018).

29. Kavanagh, G. M. \& Ross-Murphy, S. B. Rheological characterisation of polymer gels. Prog. Polym. Sci. 23, 533-562 (1998).

30. Norioka, C. Kawamura, A. \& Miyata, T. Mechanical and responsive properties of temperature-responsive gels prepared via atom transfer radical polymerization. Polym. Chem. 8, 6050-6057 (2017).

31. Sakai, T. et al. Design and fabrication of a high-strength hydrogel with ideally homogeneous network structure from tetrahedron-like macromonomers. Macromolecules 41, 5379-5384 (2008). 
32. Rivlin, R. S. \& Thomas, A. G. Rupture of rubber. I. Characteristic energy for tearing. J. Poly. Sci. 10, 291-318 (1953).

33. Tanaka, Y. et al. Determination of fracture energy of high strength double network hydrogels. J. Phys. Chem. B 109, 11559-11562 (2005).

34. Liu, C. et al. Unusual fracture behavior of slide-ring gels with movable crosslinks. ACS Macro Lett. 6, 1409-1413 (2017).
35. Xue, W., Huglin, M. B. \& Jones, T. G. J. Swelling and network parameters of crosslinked thermoreversible hydrogels of poly(N-ethylacrylamide). Eur. Polym. J. 41, 239-248 (2005).

36. Iwasaki, Y. \& Ishihara, K. Cell membrane-inspired phospholipid polymers for developing medical devices with excellent biointerfaces. Sci. Technol. Adv. Mater. 13, 064101 (2012). (14pp). 